\title{
Exact and approximate solutions for the anti-symmetric quadratic truly nonlinear oscillator
}

\author{
A. Beléndez, ${ }^{1,2}$ E. Arribas, ${ }^{3}$ C. Pascual, ${ }^{1,2}$ T. Beléndez,,${ }^{1,2}$ M. L. Alvarez,,${ }^{1,2}$ and A. Hernández ${ }^{1,2}$
}

(1) Departamento de Física, Ingeniería de Sistemas y Teoría de la Señal. Universidad de Alicante. Apartado 99. E-03080 Alicante. SPAIN

(2) Instituto Universitario de Física Aplicada a las Ciencias y las Tecnologías. Universidad de Alicante. Apartado 99. E-03080 Alicante. SPAIN

(3) Departamento de Física Aplicada. Universidad de Castilla-La Mancha. Avda. España s/n, E-02071 Albacete. SPAIN

\begin{abstract}
The exact solution of the anti-symmetric quadratic truly nonlinear oscillator is derived from the first integral of the nonlinear differential equation which governs the behaviour of this oscillator. This exact solution is expressed as a piecewise function including Jacobi elliptic cosine functions. The Fourier series expansion of the exact solution is also analyzed and its coefficients are computed numerically. We also show that these Fourier coefficients decrease rapidly and, consequently, using just a few of them provides an accurate analytical representation of the exact periodic solution. Some approximate solutions containing only two harmonics as well as a rational harmonic representation are obtained and compared with the exact solution.
\end{abstract}

Keywords: Nonlinear oscillators; Conservative systems; Truly oscillators; Fourier series expansion; Approximate solutions; Symbolic computation. 


\section{Introduction}

Nonlinear oscillator models have been widely used in many areas, not only in mechanics but also in other branches of physics, mathematics or engineering. There is a large variety of approximate methods commonly used for solving nonlinear oscillatory systems $[1,2]$. In conservative nonlinear oscillators the restoring force is not dependent on time, the total energy is constant $[2,3]$ and any oscillation is stationary. An important feature of the solutions for conservative oscillators is that they are periodic and range over a continuous interval of initial values [4]. Conservative truly nonlinear oscillatory systems are modelled by differential equations for which the restoring force has no linear approximation at $x=0$ [4]. In this paper we consider a conservative truly nonlinear oscillator for which the nonlinear function is an anti-symmetric quadratic function, $f(x)=\operatorname{sgn}(x) x^{2}$. In recent years some examples of this class of truly nonlinear oscillators have been analyzed $[4,5]$ and several techniques have been used to obtain analytical approximate solutions, such as harmonic balance, rational harmonic balance, parameter expansion, iteration or averaging methods. Recently, Cveticanin and Pogány [6] obtained an exact solution in the form of Ateb function for this class of conservative truly nonlinear oscillators. However, in the present paper we derive the exact solution of this type of oscillator and we obtain the Fourier series expansion of this exact solution. Some approximate solutions containing only two harmonics as well as a rational harmonic representation are obtained and compared with the exact solution. It may be concluded that the former are more accurate than the latter since after the second Fourier coefficient the signs of the coefficients alternate between negative and positive, whereas all the Fourier coefficients of the rational harmonic solution are positive.

\section{Formulation and solution method}

The anti-symmetric quadratic truly nonlinear oscillator is a conservative single-degree-offreedom oscillatory system governed by the following second-order differential equation

$$
\frac{\mathrm{d}^{2} x}{\mathrm{~d} t^{2}}+\operatorname{sgn}(x) x^{2}=0
$$

with initial conditions

$$
x(0)=A \quad \frac{\mathrm{d} x}{\mathrm{~d} t}(0)=0
$$


where $x$ and $t$ are the non-dimensional displacement and time. This system corresponds to a truly nonlinear oscillator [4] and is an example of a non-smooth oscillator for which $f(x)=\operatorname{sgn}(x) x^{2}$ is a non-smooth function of the non-dimensional displacement $x$. The nonlinear function $f(x)$ is also odd, i.e. $f(-x)=-f(x)$ and satisfies $x f(x)>0$ for $x \in[-A, A], x \neq 0$, where $A>0$ is the oscillation amplitude. All the solutions to the antisymmetric quadratic oscillator are periodic [4] and this system oscillates around the equilibrium position $x=0$ and the period, $T$, and periodic solution, $x$, are dependent on $A$.

The system equations are

$$
\frac{\mathrm{d} x}{\mathrm{~d} t}=y \quad \frac{\mathrm{d} y}{\mathrm{~d} t}=-\operatorname{sgn}(x) x^{2}
$$

and the differential equation for the phase-plane trajectories is

$$
\frac{\mathrm{d} y}{\mathrm{~d} x}=-\frac{\operatorname{sgn}(x) x^{2}}{y}
$$

Eq. (1) is a conservative system and has the following first integral

$$
\frac{1}{2} y^{2}+\frac{1}{3} \operatorname{sgn}(x) x^{3}=\frac{1}{3} A^{3} \geq 0
$$

which defines a family of bounded and simple closed curves in the phase-plane which are "oval" shaped as we can see in Figure 1 [4]. The $x$-axis is the "zero" null-cline and the $y$-axis is the "infinite" null-cline. These null-clines divide the phase-plane into four open regions, each coinciding with a quadrant of the plane and in each quadrant, the sign of the derivative, $\mathrm{d} y / \mathrm{d} x$, has a definite value.

Let $u=x / A$, then we can rewrite Eqs. (1) and (2) as

$$
\begin{gathered}
\frac{\mathrm{d}^{2} u}{\mathrm{~d} t^{2}}+A \operatorname{sgn}(u) u^{2}=0 \\
u(0)=1 \quad \frac{\mathrm{d} u}{\mathrm{~d} t}(0)=0
\end{gathered}
$$

Integrating Eq. (3) and using the initial conditions in Eq. (4), we arrive at

$$
\frac{1}{2}\left(\frac{\mathrm{d} u}{\mathrm{~d} t}\right)^{2}+\frac{1}{3} A \operatorname{sgn}(u) u^{3}=\frac{1}{3} A
$$


Solving for $\mathrm{d} u / \mathrm{d} t$ we obtain

$$
\frac{\mathrm{d} u}{\mathrm{~d} t}= \pm \sqrt{\frac{2 A}{3}} \sqrt{1-\operatorname{sgn}(u) u^{3}}
$$

and this can be written as

$$
\sqrt{\frac{2 A}{3}} \mathrm{~d} t= \pm \frac{\mathrm{d} u}{\sqrt{1-\operatorname{sgn}(u) u^{3}}}
$$

where the sign $( \pm)$ is chosen taking into account the sign of $\mathrm{d} u / \mathrm{d} t$ in each quadrant (Figure 1). The time it takes from point $(1,0)$ to point $(u, \mathrm{~d} u / \mathrm{d} t)$ in the lower half-plane of the phase space is

$$
t(u)=-\sqrt{\frac{3}{2 A}} \int_{1}^{u} \frac{\mathrm{d} z}{\sqrt{1-z^{3}}}
$$

The period of oscillation is four times the time taken by the oscillator to go from $u=1$ to $u=$ $0, T=4 t(0)$. Therefore

$$
T=2 \sqrt{\frac{6}{A}} \int_{0}^{1} \frac{1}{\sqrt{1-u^{3}}} \mathrm{~d} u
$$

and after some simplifications it is possible to obtain [4,7-9]

$$
T(A)=\frac{2^{1 / 6}[\Gamma(1 / 3)]^{3}}{\pi \sqrt{A}}=\frac{2^{5 / 2}}{3^{1 / 4} \sqrt{A}} K\left(\frac{2-\sqrt{3}}{4}\right)=\frac{2^{5 / 2}}{3^{3 / 4} \sqrt{A}} K\left(\frac{2+\sqrt{3}}{4}\right) \approx \frac{6.86926}{\sqrt{A}}
$$

where $\Gamma(z)$ is the Euler gamma function and $K(m)$ is the complete elliptic integral of the first kind defined as [10]

$$
K(m)=\int_{0}^{\pi / 2} \frac{\mathrm{d} \theta}{\sqrt{1-m \sin ^{2} \theta}}
$$

where $m$ is the parameter and $k \equiv \sqrt{m}$ is the elliptic modulus. From Eq. (10) we obtain $t$ as a function of $u$ for the following cases (Figure 1):

(I) Trajectory $P_{1} \rightarrow P_{2}(0 \leq t \leq T / 4$ and $1 \geq u \geq 0), u$ is positive and $\mathrm{d} u / \mathrm{d} t$ is negative:

$$
\sqrt{\frac{2 A}{3}} \int_{0}^{t} \mathrm{~d} t=-\int_{1}^{u} \frac{\mathrm{d} z}{\sqrt{1-z^{3}}}
$$


(II) Trajectory $P_{2} \rightarrow P_{3}(T / 4 \leq t \leq T / 2$ and $0 \geq u \geq-1), u$ is negative and $\mathrm{d} u / \mathrm{d} t$ is negative:

$$
\sqrt{\frac{2 A}{3}} \int_{T / 4}^{t} \mathrm{~d} t=-\int_{0}^{u} \frac{\mathrm{dz}}{\sqrt{1+z^{3}}}
$$

(III) Trajectory $P_{3} \rightarrow P_{4}(T / 2 \leq t \leq 3 T / 4$ and $-1 \leq u \leq 0), u$ is negative and $\mathrm{d} u / \mathrm{d} t$ is positive:

$$
\sqrt{\frac{2 A}{3}} \int_{T / 2}^{t} \mathrm{~d} t=\int_{-1}^{u} \frac{\mathrm{dz}}{\sqrt{1+z^{3}}}
$$

(IV) Trajectory $P_{4} \rightarrow P_{1}(3 T / 4 \leq t \leq T$ and $0 \leq u \leq 1), u$ is positive and $\mathrm{d} u / \mathrm{d} t$ is positive:

$$
\sqrt{\frac{2 A}{3}} \int_{3 T / 4}^{t} \mathrm{~d} t=\int_{0}^{u} \frac{\mathrm{dz}}{\sqrt{1-z^{3}}}
$$

Integrating Eqs. (15)-(18) we can obtain the exact solution for the anti-symmetric quadratic nonlinear oscillator. From Eq. (15) it follows for trajectory $P_{1} \rightarrow P_{2}$ :

$$
\sqrt{\frac{2 A}{3}} t=\int_{u}^{1} \frac{\mathrm{dz}}{\sqrt{1-z^{3}}}
$$

The value of the integral in Eq. (19) is [11, page 260, formula 3.139, integral 2]

$$
\int_{u}^{1} \frac{\mathrm{dz}}{\sqrt{1-z^{3}}}=\frac{1}{3^{1 / 4}} F(\beta, m)
$$

where

$$
\begin{gathered}
\beta=\arccos \left(\frac{\sqrt{3}-1+u}{\sqrt{3}+1-u}\right) \\
m=k^{2}=\sin ^{2}\left(\frac{5 \pi}{12}\right)=\frac{2+\sqrt{3}}{4}
\end{gathered}
$$

and $F(\beta, m)$ is the incomplete elliptic integral of the first kind defined as follows [10]

$$
F(\beta, m)=\int_{0}^{\beta} \frac{\mathrm{d} \theta}{\sqrt{1-m \sin ^{2} \theta}}
$$

Therefore, from Eqs. (19)-(23) it follows that

$$
\sqrt{\frac{2 A}{3}} t=\frac{1}{3^{1 / 4}} F\left(\arccos \left(\frac{\sqrt{3}-1+u}{\sqrt{3}+1-u}\right), \frac{2+\sqrt{3}}{4}\right)
$$


The inverse function of the incomplete elliptic integral of the first kind, $F^{-1}(\tau, m)$, is given by the Jacobi amplitude, $\operatorname{am}(\tau, m)[12]$

$$
F^{-1}(\tau, m)=\beta=\operatorname{am}(\tau, m)
$$

whose cosine is the Jacobi cosine function, $\operatorname{cn}(\tau, m)[12]$

$$
\cos \beta=\cos (\operatorname{am}(\tau, m))=\operatorname{cn}(\tau, m)
$$

From Eqs. (24)-(26) we can write

$$
\frac{\sqrt{3}-1+u}{\sqrt{3}+1-u}=\operatorname{cn}\left(\frac{\sqrt{2 A}}{3^{1 / 4}} t, \frac{2+\sqrt{3}}{4}\right)
$$

Solving for $x=A u$ gives

$$
x_{1}(t)=A-\sqrt{3} A\left[\frac{1-\operatorname{cn}\left(\frac{\sqrt{2 A}}{3^{1 / 4}} t, \frac{2+\sqrt{3}}{4}\right)}{1+\operatorname{cn}\left(\frac{\sqrt{2 A}}{3^{1 / 4}} t, \frac{2+\sqrt{3}}{4}\right)}\right]
$$

which is valid for trajectory $P_{1} \rightarrow P_{2}$. Eq. (28) coincides which that obtained by Mickens [4, page 33, Eq. (2.2.3)], however he does not present the values of the solution for the other trajectories in the phase-plane $\left(P_{2} \rightarrow P_{3}, P_{3} \rightarrow P_{4}\right.$ and $\left.P_{4} \rightarrow P_{1}\right)$.

From Eq. (16) it follows for trajectory $P_{2} \rightarrow P_{3}$ :

$$
\sqrt{\frac{2 A}{3}}\left(t-\frac{T}{4}\right)=-\int_{0}^{u} \frac{\mathrm{dz}}{\sqrt{1+z^{3}}}
$$

where $T$ is given by Eq. (13). Let $z=-y$, then Eq. (30) becomes

$$
\sqrt{\frac{2 A}{3}}\left(t-\frac{T}{4}\right)=\int_{0}^{-u} \frac{\mathrm{d} y}{\sqrt{1-y^{3}}}=\int_{0}^{1} \frac{\mathrm{d} y}{\sqrt{1-y^{3}}}-\int_{-u}^{1} \frac{\mathrm{d} y}{\sqrt{1-y^{3}}}
$$

The values of the two integrals on the right-hand side of Eq. (30) are

$$
\int_{0}^{1} \frac{\mathrm{d} y}{\sqrt{1-y^{3}}}=\frac{[\Gamma(1 / 3)]^{3}}{2^{4 / 3} \pi \sqrt{3}}=\frac{2}{3^{5 / 4}} K\left(\frac{2+\sqrt{3}}{4}\right)=\sqrt{\frac{2 A}{3}} \frac{T}{4}
$$




$$
\int_{-u}^{1} \frac{\mathrm{d} y}{\sqrt{1-y^{3}}}=\frac{1}{3^{1 / 4}} F\left(\arccos \left(\frac{\sqrt{3}-1-u}{\sqrt{3}+1+u}\right), \frac{2+\sqrt{3}}{4}\right)
$$

where Eq. (22) has been taken into account. Substituting Eqs. (31) and (32) into Eq. (30) gives

$$
\sqrt{\frac{2 A}{3}}\left(t-\frac{T}{2}\right)=-F\left(\arccos \left(\frac{\sqrt{3}-1-u}{\sqrt{3}+1+u}\right), \frac{2+\sqrt{3}}{4}\right)
$$

After some simplifications, the solution for $P_{2} \rightarrow P_{3}$ can be written as follows

$$
x_{2}(t)=-A+\sqrt{3} A\left[\frac{1-\operatorname{cn}\left(\frac{\sqrt{2 A}}{3^{1 / 4}}\left(t-\frac{T}{2}\right), \frac{2+\sqrt{3}}{4}\right)}{1+\operatorname{cn}\left(\frac{\sqrt{2 A}}{3^{1 / 4}}\left(t-\frac{T}{2}\right), \frac{2+\sqrt{3}}{4}\right)}\right]
$$

where we have taken into account that $\operatorname{cn}(z, m)=\operatorname{cn}(-z, m)$. We can easily verify that for trajectory $P_{3} \rightarrow P_{4}$ we obtain the same value for the solution given in Eq. (34) is obtained.

From Eq. (18) it follows for trajectory $P_{4} \rightarrow P_{1}$ :

$$
\sqrt{\frac{2 A}{3}}\left(t-\frac{3 T}{4}\right)=\int_{0}^{u} \frac{\mathrm{d} z}{\sqrt{1-z^{3}}}
$$

which can be written as follows

$$
\sqrt{\frac{2 A}{3}}\left(t-\frac{3 T}{4}\right)=\int_{0}^{1} \frac{\mathrm{d} z}{\sqrt{1-z^{3}}}-\int_{u}^{1} \frac{\mathrm{d} z}{\sqrt{1-z^{3}}}
$$

and taking into account Eqs. (24) and (31) we can finally obtain

$$
\sqrt{\frac{2 A}{3}}(t-T)=-\frac{1}{3^{1 / 4}} F\left(\arccos \left(\frac{\sqrt{3}-1+u}{\sqrt{3}+1-u}\right), \frac{2+\sqrt{3}}{4}\right)
$$

and the solution for trajectory $P_{4} \rightarrow P_{1}$ becomes

$$
x_{3}(t)=A-\sqrt{3} A\left[\frac{1-\operatorname{cn}\left(\frac{\sqrt{2 A}}{3^{1 / 4}}(t-T), \frac{2+\sqrt{3}}{4}\right)}{1+\operatorname{cn}\left(\frac{\sqrt{2 A}}{3^{1 / 4}}(t-T), \frac{2+\sqrt{3}}{4}\right)}\right]
$$


Therefore, the exact solution of Eq. (1) for the first period $(0 \leq t \leq T)$ can be written as the following piecewise function

$$
x(t)= \begin{cases}x_{1}(t), & 0 \leq t \leq \frac{T}{4} \\ x_{2}(t), & \frac{T}{4} \leq t \leq \frac{3 T}{4} \\ x_{3}(t), & \frac{3 T}{4} \leq t \leq T\end{cases}
$$

where $x(t)$ is a continuous function over the entire domain, and $x_{1}(t), x_{2}(\mathrm{t})$ and $x_{3}(\mathrm{t})$ are given in Eqs. (28), (34) and (38), respectively. Introducing the new variable $h=t / T$ and taking into account that $u=x / A$, we can re-write Eqs. (28), (34), (38) and (39) as follows

$$
u(t)= \begin{cases}u_{1}(h), & 0 \leq h \leq \frac{1}{4} \\ u_{2}(h), & \frac{1}{4} \leq h \leq \frac{3}{4} \\ u_{3}(h), & \frac{3}{4} \leq h \leq 1\end{cases}
$$

where

$$
\begin{gathered}
u_{1}(h)=1-\sqrt{3}\left[\frac{1-\operatorname{cn}(M h, m)}{1+\operatorname{cn}(M h, m)}\right] \\
u_{2}(h)=-1+\sqrt{3}\left[\frac{1-\operatorname{cn}\left(M\left(h-\frac{1}{2}\right), m\right)}{1+\operatorname{cn}\left(M\left(h-\frac{1}{2}\right), m\right)}\right] \\
u_{3}(h)=1-\sqrt{3}\left[\frac{1-\operatorname{cn}(M(h-1), m)}{1+\operatorname{cn}(M(h-1), m)}\right]
\end{gathered}
$$

In these equations, $m=k^{2}=\frac{2+\sqrt{3}}{4} \approx 0.933013$ (see Eq. (22)), and parameter $M$ is defined as (see Eq. (13))

$$
M=\frac{\sqrt{2 A}}{3^{1 / 4}} T(A)=\frac{8}{3} K\left(\frac{2+\sqrt{3}}{4}\right) \approx 7.3815
$$

Eqs. (40)-(43) can be easily generalized for any period, $p$, as follows 


$$
u^{(p)}(h)= \begin{cases}u_{1}^{(p)}(h), & p-1 \leq h \leq p-\frac{1}{4} \\ u_{2}^{(p)}(h), & p-\frac{1}{4} \leq h \leq p-\frac{3}{4} \\ u_{3}^{(p)}(h), & p-\frac{3}{4} \leq h \leq p\end{cases}
$$

where

$$
\begin{gathered}
u_{1}^{(p)}(h)=1-\sqrt{3}\left[\frac{1-\operatorname{cn}(M(h-p+1), m)}{1+\operatorname{cn}(M(h-p+1), m)}\right] \\
u_{2}^{(p)}(h)=-1+\sqrt{3}\left[\frac{1-\operatorname{cn}\left(M\left(h-p+\frac{1}{2}\right), m\right)}{1+\operatorname{cn}\left(M\left(h-p+\frac{1}{2}\right), m\right)}\right] \\
u_{3}^{(p)}(h)=1-\sqrt{3}\left[\frac{1-\operatorname{cn}(M(h-p), m)}{1+\operatorname{cn}(M(h-p), m)}\right]
\end{gathered}
$$

where $p=1,2,3, \ldots$ is related to the first-, second-, third-, ..., period, respectively. Comparing Eqs. (40)-(43) and Eqs. (45)-(48) we can see that $u(h) \equiv u^{(0)}(h), u_{1}(h) \equiv u_{1}^{(0)}(h)$, $u_{2}(h) \equiv u_{2}^{(0)}(h)$, and $u_{3}(h) \equiv u_{3}^{(0)}(h)$. Figure 2 gives the periodic solution $u(h)$ for the time of one period. This figure was plotted using Eqs. (40)-(43) in their respective regions.

\section{Fourier series expansion of the exact solution}

Since the nonlinear function $\operatorname{sgn}(u) u^{2}$ is an odd function of $u$, the periodic solution $u(h)$ in Eq. (40) can be represented by a Fourier series containing only odd multiples of $2 \pi h$, i.e.

$$
u(h)=\sum_{n=0}^{\infty} a_{2 n+1} \cos [(2 n+1) 2 \pi h]
$$

where

$$
a_{2 n+1}=2 \int_{0}^{1} u(h) \cos [(2 n+1) 2 \pi h] \mathrm{d} h=8 \int_{0}^{1 / 4} u_{1}(h) \cos [(2 n+1) 2 \pi h] \mathrm{d} h
$$

This equation allows us to obtain the values of the coefficients $a_{2 n+1}$ of the Fourier series expansion of the exact periodic solution $u(h)$. The integral in Eq. (50) must be computed 
numerically and this was done with the help of symbolic computation software such as MATHEMATICA.

We computed some of the coefficients of the Fourier series expansion of the exact solution and their values are shown in Table 1. From this table it may be seen that the Fourier coefficients decrease rapidly and, consequently, the use of just a few terms in the Fourier series expansion in Eq. (49) provides an accurate analytical representation of the exact periodic solution in Eq. (40). The first two Fourier coefficients are positive and from the second Fourier coefficient on, the signs of the coefficients alternate between negative and positive. We also calculated the ratio of neighbouring coefficients and the results are shown in Table 2. The first two ratios indicate the dominance of the lower harmonics in the periodic solution. In fact, from Tables 1 and 2 we conclude that using only two harmonics, it would be possible to construct an accurate analytical approximate solution to Eq. (1), as we shall see in the next section.

\section{Some analytical approximate solutions}

In this section, we present some simple analytical approximate solutions for the system described in Eq. (1). As the exact solution has an infinite number of harmonics (see Eq. (49)), we can truncate this series expansion considering analytical approximate solutions with a finite number of harmonics. From Tables 1 and 2 we conclude that there are two dominant harmonics, $a_{1}$ and $a_{3}$, and therefore consider only the cases in which one or two harmonics are present in the approximate solution, which correspond to the first and the second approximation, respectively. In order to have a global estimation of the accuracy of each approximate solution, $L_{2}$ norm errors were calculated as follows [13]

$$
L_{2}=\sqrt{\int_{0}^{1}\left|u(h)-u_{a p p}(h)\right|^{2} \mathrm{~d} h}
$$

where $u(h)$ is the exact solution given in Eq. (40) and $u_{a p p}(h)$ is the approximate solution. We assume that the period of all approximate solutions is the exact period given in Eq. (13).

In the first approximation of motion, only one harmonic is considered. This is the simplest analytical approximate solution and can be written as

$$
u_{a 1}(h)=\cos (2 \pi h)
$$


where the amplitude satisfies the first of the initial conditions in Eq. (7). For this approximate solution the value of the " $L_{2}$ error" is $L_{2}=0.025467$.

When the first two harmonics are considered, the solution corresponds to the second approximation. We consider three different cases. In the first, we assume that the amplitude for the first harmonic is the first coefficient (Table 1, $a_{1}=0.974801$ ) in the Fourier series expansion given in Eq. (49) and the amplitude for the second harmonic is obtained taking into account the first of the initial conditions in Eq. (7). Then, we can write this second approximation as follows

$$
u_{b 2}(h)=a_{1} \cos (2 \pi h)+\left(1-a_{1}\right) \cos (6 \pi h)
$$

for which the amplitude of the second harmonic is 0.025199 . The value of the " $L_{2}$ error" for this approximate solution is $L_{2}=0.00059425$.

A second approximate solution can be built up assuming that the amplitude for the second harmonic is the second coefficient (Table $1, a_{3}=0.025724$ ) in the Fourier series expansion given in Eq. (49) and the amplitude for the first harmonic is obtained taking into account the first of the initial conditions in Eq. (7). This corresponds to the equation

$$
u_{c 2}(h)=\left(1-a_{3}\right) \cos (2 \pi h)+a_{3} \cos (6 \pi h)
$$

Now the amplitude of the first harmonic is 0.974276 and $L_{2}=0.00059425$. This means that Eqs. (53) and (54) give identical results.

In order to obtain a better second analytical approximate solution we consider the following equation

$$
u_{d 2}(h)=q \cos (2 \pi h)+(1-q) \cos (6 \pi h)
$$

where $0<|q|<1$. The optimum value for $q$ is calculated by minimizing the $L_{2}$-norm. In Figure 3 we plotted the $L_{2}$-norm as a function of $q$ for the approximate solution given in Eq. (55). The minimum value of $L_{2}$ is 0.00053322 which is obtained when $q=0.974539$. We can conclude that the second approximate solution given in Eq. (55) with $q=0.974539$ (1-q= 0.025461) is slightly better than the approximate solutions given in Eqs. (54)-(55). As can be seen, in the last approximation, neither the amplitude of the first harmonic nor that of the second one coincides with the first or the second exact Fourier coefficients, respectively. However, this approximate solution is the most accurate. 
Finally, it was shown that a rational harmonic representation provides an excellent approximation to the solution of conservative nonlinear oscillatory systems with high accuracy [2,5,14]. This rational representation not only provides accurate results but also approximates all the harmonics of the exact solution of a given differential equation. Taking this into account, we consider the following approximation to the exact solution of Eq. (1) [13]

$$
u_{e 2}(h)=\frac{\left(1-r^{2}\right) \cos (2 \pi h)}{1+r^{2}-2 r \cos (4 \pi h)}
$$

where $0<|r|<1$. Eq. (56) satisfies the initial conditions in Eq. (7) independently of parameter $r$. The optimum value for $r$ is also calculated by minimizing the $L_{2}$-norm and in Figure 4 we plotted the $L_{2}$-norm as a function of $r$ for the approximate solution given in Eq. (56). The minimum value of $L_{2}$ is 0.0010905 which is obtained when $r=0.025745$. As can be seen, even though the rational approximation in Eq. (56) approximates all the harmonics of the solution, this approximation is not as accurate as that given in Eq. (55), which only contains the first two harmonics.

For comparison, in Figure 5 we present the differences between normalized exact and approximate solutions, $\Delta=u-u_{a p p}$, as a function of $h$, for the four approximate solutions considered previously.

At this point it is necessary to ask the following question: why does the rational harmonic representation in Eq. (56) not give as accurate results as the two-harmonics representation in Eq. (55)? In order to answer this question, we firstly consider the Fourier series expansion of the rational harmonic solution given in Eq. (56), which can be written as follows

$$
u_{e 2}(h)=\sum_{n=0}^{\infty} e_{2 n+1} \cos [(2 n+1) 2 \pi h]
$$

The general expression of the coefficients of this Fourier series expansion was calculated as follows [13]

$$
e_{2 n+1}=(1-r) r^{n}
$$

For $r=0.025745$, the first two coefficients are $e_{1}=0.974255$ and $e_{3}=0.0250822$, respectively. As we obtained $0<r<1$, from Eq. (58) it follows that all the coefficients of the Fourier series expansion of Eq. (56) are positive, i.e., $e_{2 n+1}>0(n=0,1,2, \ldots)$. However, from Table 1 we can see that from the second Fourier coefficient, $a_{3}$, on the signs of the 
Fourier coefficients alternate between negative and positive $\left(a_{1}>0, a_{3}>0, a_{5}<0, a_{7}>0, a_{9}\right.$ $\left.<0, a_{11}>0, \ldots\right)$. Thus, the rational harmonic representation in Eq. (56) does not approximate correctly all the harmonics of the exact solution. From Eqs. (49) and (50) it follows that

$$
\sum_{n=2}^{\infty} a_{2 n+1}=-0.00052462<0
$$

However, substituting $r=0.025745$ in Eqs. (57) and (58) gives

$$
\sum_{n=2}^{\infty} e_{2 n+1}=0.00066281>0
$$

From the above it may be concluded that, for $0<r<1$, the rational harmonic representation in Eq. (56) will give more accurate results than the two-harmonic representation when all the coefficients of the Fourier series expansion of the exact solution of a conservative nonlinear oscillator were positive. However, this affirmation needs further research.

\section{Conclusions}

The exact solution of the anti-symmetric quadratic truly nonlinear oscillator was obtained as a piecewise function involving Jacobi elliptic functions and this solution was valid for all periods of the motion. This function is therefore more complete than the equation obtained by Mickens [4], which is only valid for the first quarter of the first oscillation period. The Fourier series expansion of the exact solution was numerically calculated and it was possible to draw two conclusions. First, the Fourier coefficients decrease rapidly and, consequently, the use of just a few terms in the Fourier series expansion provides an accurate analytical representation of the exact periodic solution, and second, the signs of the Fourier coefficients of the exact solution, from the second coefficient on, alternate between negative and positive. This treatment could be applied to analyse of observed data relies on nonlinear dynamics [15]. Some simple approximate solutions containing only two harmonics were analyzed and discussed and the best of them was calculated. In addition, an approximate rational harmonic representation was obtained and, surprisingly, even though this rational representation approximates all the harmonics of the exact solution, it does not give as accurate results as the two-harmonic approximate solution. 


\section{Acknowledgements}

This work was supported by the "Generalitat Valenciana" of Spain (projects PROMETEO/2011/021 and ISIC/2012/013), and by the "Vicerrectorado de Tecnologías de la Información" of the University of Alicante, Spain (project GITE-09006-UA). 


\section{References}

[1] A. H. Nayfeh, Problems in Perturbation, John Wiley \& Sons, New York, 1985.

[2] R. E. Mickens, Oscillations in Planar Dynamics Systems, World Scientific, Singapore, 1996.

[3] A. H. Nayfeh and D. T. Mook, Nonlinear Oscillations, Wiley-Interscience, New York, 1979.

[4] R. E. Mickens, Truly Nonlinear Oscillations, World Scientific, Singapore, 2010.

[5] A. Beléndez, E. Gimeno, M. L. Alvarez, M. S. Yebra and D. I. Méndez, "Analytical approximate solutions for conservative nonlinear oscillators by modified rational harmonic balance method," International Journal of Computer Mathematics, vol. 87, no. 7, pp. 1497-1511, 2010. DOI: 10.1080/00207160802380942.

[6] L. Cveticanin and T. Pogány, "Oscillator with a sum of noninteger-order nonlinearities," Journal of Applied Mathematics, vol. 2012, Art. ID 649050, 20 pages 2012. DOI: $10.1155 / 2012 / 649050$.

[7] E. T. Whittaker and G. N. Watson, A Course of Modern Analysis. Cambridge, Cambridge University Press, 1996. Chapter 22.

[8] E. W. Weisstein, "Elliptic Integral Singular Value.” From MathWorld-A Wolfram Web Resource. http://mathworld.wolfram.com/EllipticIntegralSingularValue.html

[9] E. W. Weisstein, "Elliptic Integral Singular Value--k_3." From MathWorld-A Wolfram Web Resource.

http://mathworld.wolfram.com/EllipticIntegralSingularValuek3.html

[10] L. M. Milne-Thomson, "Elliptic integrals", Ch. 17 in Handbook of Mathematical functions with Formulas, Graphics and Mathematical Tables. M. Abramowitz and I. A. Stegun (Eds.). New York, Dover, pp. 587-607, 1972.

[11] I. S. Gradshteyn and I. M. Ryzhik, Table of Integrals, Series, and Products, $6^{\text {th }}$ Edition. A. Jeffrey and D. Zwillinger (Eds.). Academic Press, 2000. 
[12] L. M. Milne-Thomson, "Jacobian elliptic functions and theta functions", Ch. 16 in Handbook of Mathematical functions with Formulas, Graphics and Mathematical Tables. M. Abramowitz and I. A. Stegun (Eds.). New York, Dover, pp. 567-581, 1972.

[13] M. Febbo, “A finite extensibility nonlinear oscillator," Applied Mathematics and Computation, vol. 217, no. 14, pp. 6464-6475, 2011. DOI: 10.1016/j.amc.2011.01.011

[14] S. B. Yamgoué, J. R. Bogning, A. K. Jiotsa and T. C. Kofané, "Rational harmonic balance-based approximate solutions to nonlinear single-degree-of-freedom oscillator equations," Physica Scripta, vol. 81, no. 3, Art. 035003 (6 pp), 2010. DOI: 10.1088/0031-8949/81/03/035003

[15] S. Kodba, M. Perc and M. Marhl, "Detecting chaos from a time series," European Journal of Physics, vol. 26, no. 1, pp. 205-215, 2005. DOI: 10.1088/01430807/26/1/021 


\section{FIGURE CAPTIONS}

Figure 1.- Trajectories in the phase plane for the anti-symmetric quadratic nonlinear oscillator.

Figure 2.- Plot of the exact solution for the first period for the anti-symmetric quadratic nonlinear oscillator.

Figure 3.- $L_{2}$ norm as a function of $q$ for the approximate solution given in Eq. (55).

Figure 4.- $L_{2}$ norm as a function of $r$ for the approximate solution given in Eq. (56).

Figure 5.- Differences between normalized exact (Eq. (40)) and approximate solutions, $\Delta=u-u_{a p p}$, as a function of $h$, for the four approximate solutions considered (circles, Eq. (53); triangles, Eq. (54); squares, Eq. (55); rhombus, Eq. (56)).

\section{TABLES}

Table 1. Values for the first eight coefficients of the Fourier series expansion of the exact solution.

Table 2. Ratios of each pair of neighbouring Fourier coefficients. 
FIGURE 1

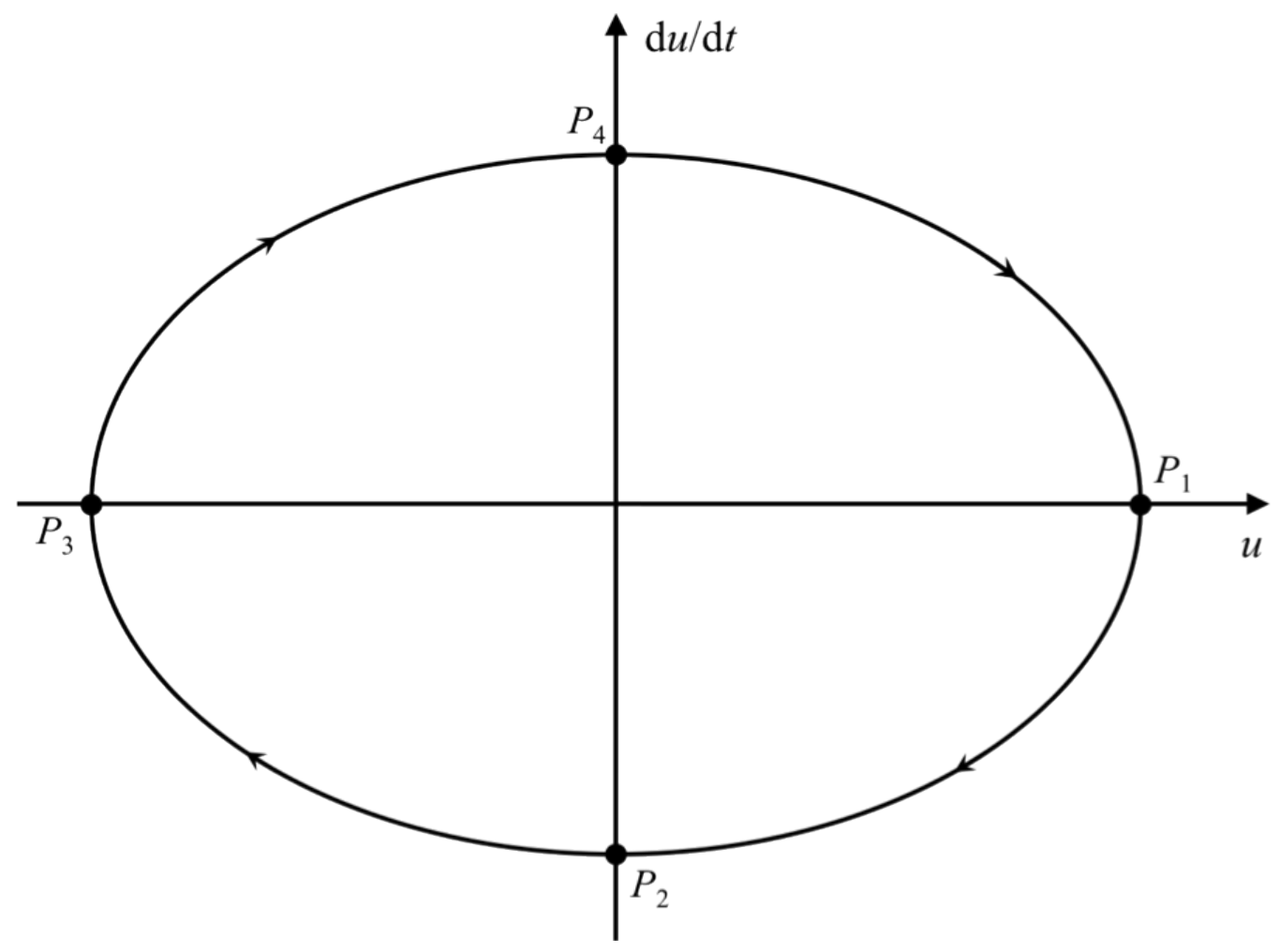


FIGURE 2

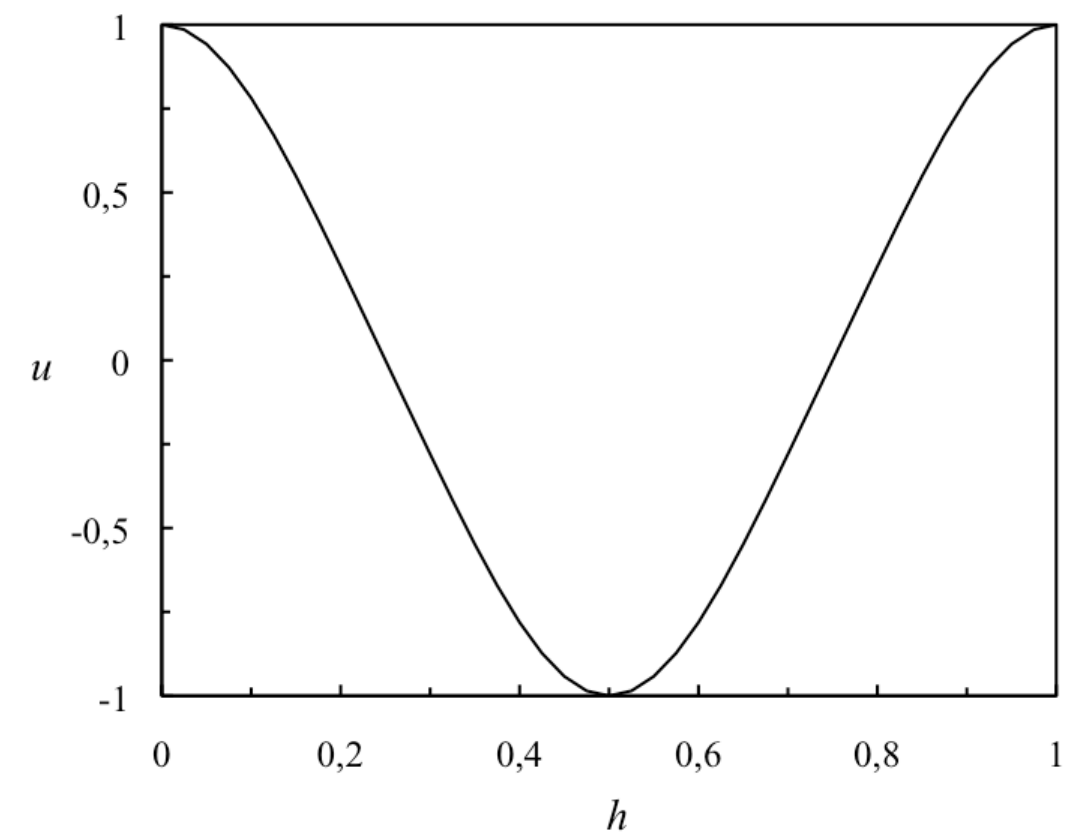


FIGURE 3

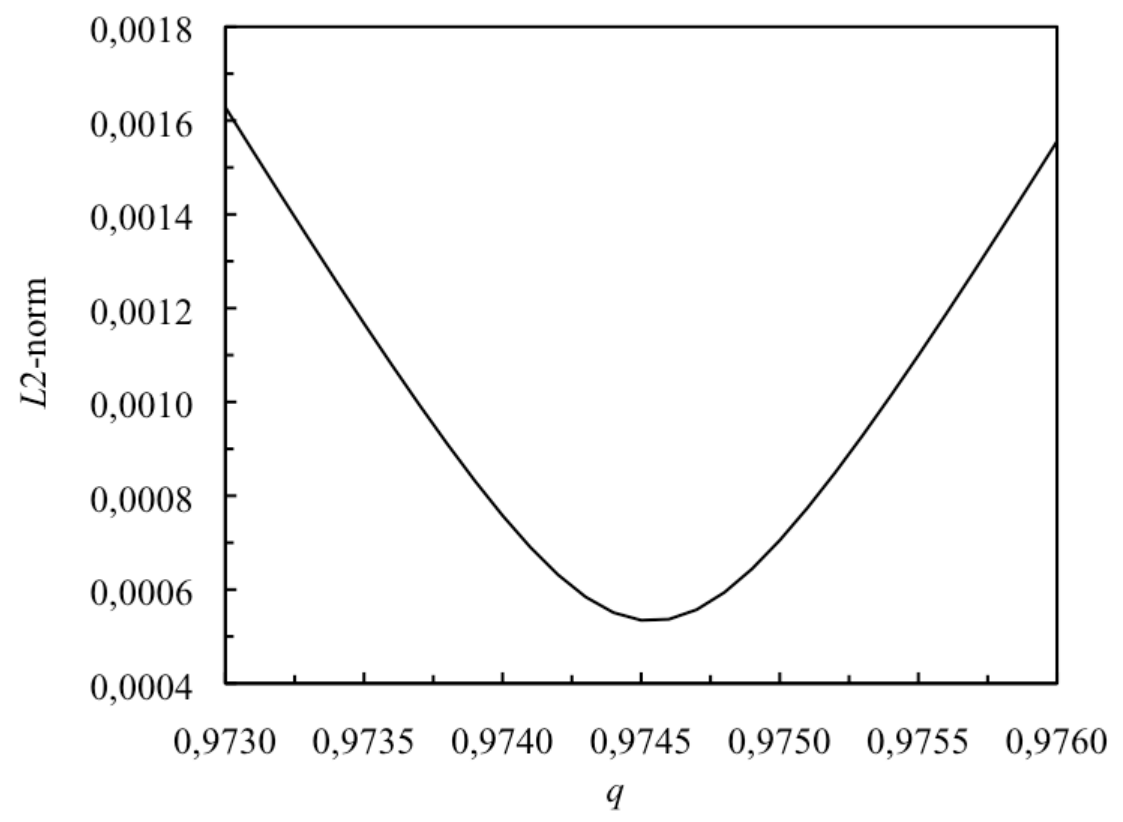


FIGURE 4

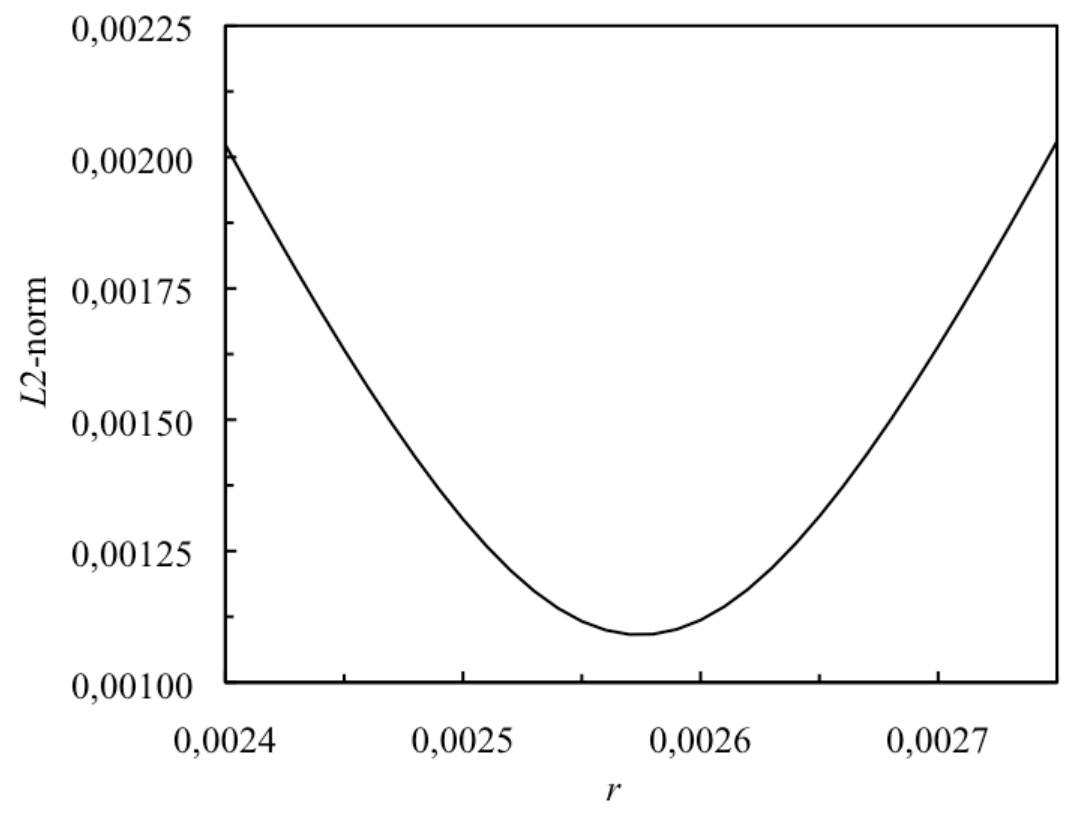


FIGURE 5

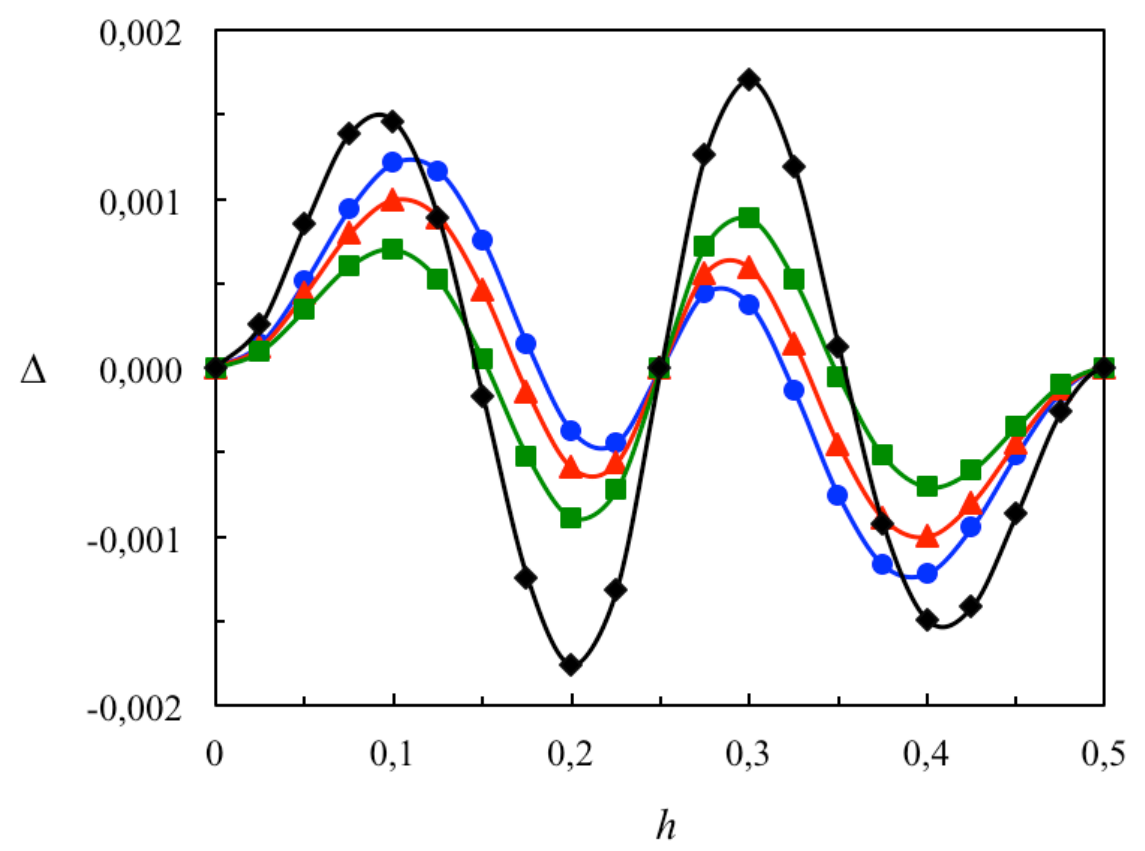


Table 1.

\begin{tabular}{|l|l|l|l|}
\hline$a_{1}$ & 0.974801 & $a_{9}$ & -0.0000410429 \\
\hline$a_{3}$ & 0.0257238 & $a_{11}$ & 0.0000150565 \\
\hline$a_{5}$ & -0.000638861 & $a_{13}$ & -0.00000653164 \\
\hline$a_{7}$ & 0.000144662 & $a_{15}$ & 0.00000319375 \\
\hline
\end{tabular}


Table 2.

\begin{tabular}{|l|l|l|l|}
\hline$a_{3} / a_{1}$ & 0.0263887 & $a_{11} / a_{9}$ & -0.366848 \\
\hline$a_{5} / a_{3}$ & -0.0248354 & $a_{13} / a_{11}$ & -0.433809 \\
\hline$a_{7} / a_{5}$ & -0.226438 & $a_{15} / a_{13}$ & -0.488965 \\
\hline$a_{9} / a_{7}$ & -0.283715 & $a_{17} / a_{15}$ & -0.534833 \\
\hline
\end{tabular}

